\title{
The Value of Fabry-Perot Interferometry in Studying Long-Term Convective Line Shifts
}

\author{
Robert S. McMillan, T. H. Bressi, J. L. Montani, T. L. Moore, M. L.
} Perry and A. F. Tubbiolo

Lunar and Planetary Laboratory, University of Arizona, Tucson, AZ 85721

\begin{abstract}
Small $<10 \mathrm{~m} \mathrm{~s}^{-1}$ variations of radial velocity (RV) with multi-year periods in solar-type stars may be indistinguishable from the effects induced on lines by stellar activity cycles (Dravins 1985; Saar $\&$ Donahue 1997). Dravins (1992) recommended a resolving power $R>$ $3 \times 10^{5}$ to measure accurately the subtle changes in the shapes of bisectors of photospheric absorption lines driven by changes of granular convection in slowly rotating dwarf stars. Butler et al. (1996) measure impressively small amplitudes of RVs by using echelle spectrographs that cover a broad spectrum. However, to cover a broad spectrum the resolving power is typically limited to $<7 \times 10^{4}$, and the necessary presence of the iodine absorption spectrum may make it difficult to measure convective line shifts contemporaneously with the RV time series. Furthermore, to reach an RV accuracy of $\pm 3 \mathrm{~ms}^{-1}$ the whole profile of each line is used, thus maximizing the possibility that changes in the shapes of the lines' Cbisectors could induce an apparent variation of $\mathrm{RV}$.

Dravins (1985) recommended the exclusive use of the steep flanks of photospheric absorption lines to minimize the effects of convection on apparent RV. McMillan et al. $(1993,1994)$ demonstrated that such RV measurements made with a Fabry-Perot etalon (FPE) interferometer in transmission can be stable in the presence of stellar line variations seen by other investigators whose measurements were not based exclusively on line flanks. Dravins also prescribed high resolving power, high signalto-noise ratio, high instrumental contrast, and low instrumental wings to analyze the rest of the line profile for convectively-driven changes (Dravins $1978,1987,1992)$. A double- or multiple-pass FPE scanning whole line profiles can provide high $\mathrm{R}$, high contrast, low wings, and a stable, symmetrical line spread function with small (portable) optics, although the low photon efficiency will restrict its use to a few carefully selected stars. The spectral classes of these stars should span the spectral classes of the stars being monitored by others for planets. We describe a possible implementation of this concept that has the potential for adequate photon flux: observing symbiotically through another instrument on a $6-\mathrm{m}$ to 10 -m class telescope.
\end{abstract}




\section{Purpose and Background}

Our goal is to support the searches for planetary systems like our own. In the early 1980s we developed a technique sensitive enough to look for small, slow changes of stellar RVs induced by the orbital motion of planets about solar-type stars. Later, other groups, by surveying large numbers of stars, made exciting discoveries of substellar companions to some such stars (Latham et al. 1989; Mayor \& Queloz 1995; Mazeh, Latham, \& Stefanik 1996; Marcy \& Butler 1996; Butler \& Marcy 1996; Noyes et al. 1997; Cochran et al. 1997). With the notable exceptions of 47 UMa (Butler \& Marcy 1996) and 14 Her (Mayor et al. 1998), the small orbits of these companions are uncharacteristic of our solar system and seem to exist around only a few per cent of the stars surveyed (Mazeh et al. 1996, Marcy \& Butler 1996).

Around the rest of the stars the frequency of occurrence and detailed character of planetary systems, with much smaller RV amplitudes and much longer periods, are still unknown and will require more exacting work to be revealed. This requirement also applies to planets in larger orbits about the stars that have already been found to have short-period companions. Three reasons why it is more difficult to use RVs to detect planetary systems like ours are that (i) the amplitude of variation is small, (ii) the periods are long, and (iii) there is an alternative interpretation for small, slow variations of apparent $R V$. This is due to the similarity of time scales of planetary orbits and stellar activity ("spot") cycles (Wilson 1978), combined with the fact that changes of stellar magnetic activity can alter the apparent RV of stellar absorption lines. Toward better understanding of the magnitude of this effect, we established a new upper limit on the variability of the RV of the integrated disk of the Sun as measured from the steep flanks of photospheric absorption lines in violet light (McMillan et al. 1993). However, that result was on only one star, observed for only 5 years, in a specialized way that is least expected to be influenced by convective line shifts. Much more work needs to be done to understand the effects of stellar activity on apparent $\mathrm{RVs}$.

The effect of stellar activity on line profiles was predicted qualitatively by Dravins $(1985,1992)$ and quantitatively by Saar \& Donahue (1997). Time dependences of line bisectors have been observed. Gray \& Baliunas (1995) observed a 5-year cyclic variation of line bisectors in $\epsilon$ Eri. Gray et al. (1996) showed the time dependence of line bisectors of $\xi$ Boo A through variations of chromospheric activity. However, there have not been many measurements of the effect of line bisector changes on apparent RV. Comparison of the work of McMillan et al. (1993) and Deming \& Plymate (1994) on the Sun shows the sensitivity of such results to the properties of the lines and how the lines are measured. Saar, Butler, \& Marcy (1998) show correlations of the residual dispersion of RVs of 17 stars with stellar temperature, Ca II HK emission, and rotational speed. The monumental work of Livingston (1998) based on 18 years of extremely high resolution spectroscopy of the Sun is eagerly awaited.

In the search for "Jupiters", avoiding stars with active chromospheres will severely limit the region of the HR diagram being explored, and at small amplitudes all stars exhibit activity or variability. The Sun has an activity cycle and shows variations of line asymmetries and other properties of lines (Livingston 1987, 1991; Deming \& Plymate 1994; Gray \& Livingston 1997), but if observed 
from afar its chromosphere would be considered stable compared to some stars in the lists of targets of RV programs. In particular, dwarf stars cooler than the Sun are numerous among the nearest stars, and chromospheric activity is common among such stars. Therefore RVs of astrometric targets are especially vulnerable to changing convection in the stars' atmospheres. A long range goal of the RV community should be to acquire the ability to measure planetary orbits in the presence of convective line shifts. This will require more thorough understanding derived from carefully made observations.

\section{Technical Approach}

Dravins (1992) argues that a resolving power $R=\lambda / \Delta \lambda>3 \times 10^{5}$ is required to represent accurately the shapes of line bisectors. To be sure, changes of large enough amplitude or on strong lines can be detected with $\mathrm{R}$ as "low" as $5 \times 10^{4}$, but we are seeking high sensitivity to bisector changes in the unselected, moderate-to-weak lines that contribute most to RV information, and we want true bisector shapes to make it possible to compare the data with models. If $\mathrm{R}$ $=3 \times 10^{5}$ seems extravagant, realize that is $1 \mathrm{~km} \mathrm{~s}^{-1}$ resolution and the Sun rotates with an equatorial surface speed of only $2 \mathrm{~km} \mathrm{~s}^{-1}$. There are also other line broadening mechanisms with similar widths, and to deconvolve them from each other and from the instrumental broadening function, the latter needs to be not only narrow but stable, smooth, and symmetric.

Resolving power this high is rare in stellar spectroscopy, and even rarer with low enough wings to provide the true shapes of line profiles (Dravins 1978, 1987). Fourier Transform Spectrometers, double-pass echelle spectrographs with an intermediate slit, and multiple-pass FPEs can provide the degree of spectral purity required. The multiplexed FTS developed by Nordgren \& Hajian (1999) may be especially effective for this type of work. It is unclear whether a singlepass echelle spectrograph designed for use with adaptive optics (Ge et al. 1997), with its nominally high resolving power, has high enough contrast for this type of work. All instruments in this class sacrifice limiting magnitude for resolving power and spectral purity. Long integration times on large telescopes will be the rule if typical planet-search targets are to be studied.

FPEs can provide an extremely high $\mathrm{R}$ with relatively small optics (Hobbs 1969; Vaughan 1989). Resolving powers exceeding our requirements have been achieved with FPEs as early as the original one by Perot \& Fabry (1899), which Vaughan (1989) points out had $\mathrm{R}=10^{6}$. An FPE interferometer with an orderseparating spectrograph provides high stability of both the wavelength scale and the wavelength broadening function. The latter is symmetric and insensitive to focus, alignment of the spectrograph, non-flatness of the CCD, or imperfect charge transfer efficiency of the CCD. CCD pixel position is not mapped into RVs and many CCD pixels are integrated into each flux measurement (McMillan et al. $1985,1986,1990,1993,1994)$. With enough photon flux, the complete shapes of line profiles can be measured by taking measurements at a sequence of tuned wavelengths. However, the way we had been using an FPE also allows the effects of convective line shift on RV to be minimized. Dravins (1985) recommended the steep flanks of photospheric absorption lines to minimize the effects of convection on apparent RV. RV measurements made in this way with a FPE interferometer 
were stable in the presence of stellar line variations seen by other investigators whose measurements were not based exclusively on line flanks (McMillan et al. $1993,1994)$.

To get enough light for $\mathrm{R}=3 \times 10^{5}$, we have been experimenting with a new way of observing, inspired by our realization that astrometric observations of nearby stars, while integrating on the faint reference star fields, must necessarily reject most of the light of the target star in the center of the field in order to avoid detector saturation. In the case of Gatewood's $(1987,1996)$ Multichannel Astrometric Photometer (MAP) at the Allegheny Observatory, the light from each star in the field is delivered away from the focal plane by a separate optical fiber. The required attenuation of the light from the central star is accomplished at the output end of the target star's fiber. This ratio is typically 1 per cent for astrometry and 99 per cent rejected for most of Gatewood's astrometric targets, so he has agreed to let us use the rejected light for simultaneous spectroscopy. Our combined instruments are collectively referred to as the MAPS: Multichannel Astrometric Photometer with Spectrograph.

We refocus the light reflected by the target star's beamsplitter into a second optical fiber as shown by Fig. 2 of Gatewood et al. (1997), the output of which is the entrance aperture to our instrument. Since the image scrambling by an optical fiber has always been a fundamental part of our technique for accurate measurement of RVs (McMillan et al. 1988), and the FPE intrinsically can provide high resolving power with a larger entrance aperture (fiber output) than that with a grating spectrograph, our technique is naturally suited to this mode of "symbiotic" observing. This very high resolution spectroscopy will complement the programs of Cochran \& Hatzes (1994) at McDonald Observatory $\left(\mathrm{R}=2.2 \times 10^{5}\right)$, Marcy \& Butler $(1996)$ at Lick $\left(\mathrm{R}=1.2 \times 10^{5}\right)$; Butler et al. 1996) and at Keck $\left(\mathrm{R}=6.7 \times 10^{4}\right.$; Vogt 1992), Gray et al. (1996) at the University of Western Ontario $\left(R=10^{5}\right)$, Brown et al. (1994) at Mt. Hopkins $\left(\mathrm{R}=7 \times 10^{4}\right)$, and Mayor \& Queloz at Haute Provence $\left(\mathrm{R}=4.2 \times 10^{4}\right.$; Baranne et al. 1996).

The integration time needed to measure line bisectors at $\mathrm{R}=3 \times 10^{5}$ with a scanning FPE can be estimated for the required ratio of signal to noise of $10^{3}$ (Dravins 1978, 1987, 1992) if we assume a star of $V=5$ mag observed with a 6 - $\mathrm{m}$ to $10-\mathrm{m}$ class telescope. On the $10-\mathrm{m}$ Keck II we observe through Gatewood's Ronchi ruling, which cuts the time-averaged flux from the star in half. This is equivalent to our observing without the MAPS on the 6.5-m MMT, so we will assume a $6.5-\mathrm{m}$ telescope aperture. Ten tilt settings in $0.5 \mathrm{~km} \mathrm{~s}^{-1}$ steps, scanned repeatedly and rapidly, should take about two hours in green light. Stable guiding, seeing, and sky transparency will be necessary for scanned profiles to be accurate, but the alternative of spanning the line profile with detector pixels would probably not provide the requisite uniformity of response of one part in a few thousand. Twelve nights per year with an average of three observations per night (allowing for weather) would provide three line profiles per year on each of 12 carefully selected stars.

The instrument we built in 1997 is shown schematically in Fig. 7 of Gatewood et al. (1997). The interferometer part consists of the fiber output termination and its mount, the collimating lens, the etalon, its tilt mechanism, and a vibration-damped surface inside a temperature-controlled box on the table. The 
spectrograph is merely used to separate the interference orders and distribute them on a CCD. Wavelength and RV information are derived from knowledge of the thickness of the etalon and its tilt, not the placement or resolution of the spectrum on the CCD.

\section{Progress}

We had observing runs with the MAPS on the Keck II Telescope in 1997 to experiment with the concept of symbiotic observing. Due to the late arrival of our funds in 1997, an FPE interferometer was not available, but the spectrographic part of the configuration was ready for tests of throughput. The performance with an FPE added to this system could be extrapolated from these tests.

We planned our observing with a healthy respect for the physical stress of setting up and conducting a scientific experiment in the oxygen-deprived conditions of Mauna Kea. We tested the spectrograph and optical fiber at LPL on starlight before shipment, allowing us to develop our observing procedures in the comfort of our own lab. McMillan made two trips to Mauna Kea with Gatewood in advance of the spectroscopic run. On the first trip Gatewood and McMillan simply conferred with Keck personnel, visited the site and took plenty of photos and video of the areas in the Keck II building we would be using. On the second trip McMillan helped Gatewood and his personnel observe with the astrometric instrument without the spectrograph. We saw how spectrographic operations could mesh with theirs with minimal interference and to what extent Keck personnel were available to assist. We then shipped all our equipment and tools weeks in advance of the first spectroscopic run to permit Keck personnel plenty of time to truck our crates to the summit. To minimize the burden on Keck personnel and resources we rented our own four-wheel drive vehicle to commute between the Hale Pohaku residence and the summit, and staffed each of our spectroscopic observing runs with three people from our group. We checked into the residence about ten days in advance of the first observing night, so as to get acclimated to the summit conditions while gradually unpacking and setting up the experiment. These measures were quite expensive but would have been worthwhile if we had been allowed to leave our instrument set up between observing runs (see below). We brought all our own computers, accessories, tools, materials and spare parts to make our daily operations as self-sufficient as possible. A thermoelectrically cooled CCD was used so we would not have to rely on a supply of liquid nitrogen from Keck personnel. We found a place to set up our spectrograph and computers that was out of the general workaday traffic in the building.

We enjoyed hospitality and cooperation from the summit staff of the Keck Observatory, who enthusiastically performed those tasks that we were not authorized to do. Our equipment was transported from our shipping destination (Keck Headquarters in Waimea) to the summit well in advance of our observing run, and was handled with care. A long, low-OH fused silica fiber of $1 \mathrm{~mm}$ core diameter made by $3 \mathrm{M}$ with SMA connectors was pulled by Keck personnel through the Keck II elevation cable wrap from the straight Cassegrain focus to the only possible location for the spectrograph table: the basement. This location was much farther away from the telescope focus than we had assumed, 
so the fiber was much longer $(110 \mathrm{~m})$ than we would have liked. Nevertheless, the fiber manufacturer predicted absorption losses of only 10 per cent longward of $700 \mathrm{~nm}$ with this fiber, not including losses due to focal ratio mismatch and end reflections. Our measurements of throughput made with a Th-Ne hollowcathode lamp and a single-channel silicon photodiode confirmed this prediction, within the likely systematic errors of the crude field setup.

All our spectrographic equipment was functional well in advance of the first night and operated reliably on all nights scheduled. Spectra of several of Gatewood's brighter targets were obtained. Extrapolation of those results to that expected if an etalon were added to the instrument indicated that the experiment would have worked at the Keck II telescope and that there was considerable room for improvement in throughput if a smaller spectrograph could be built and placed in proximity to Gatewood's instrument, at the end of a short optical fiber. This gain will be achieved mostly by moving the observations from the near-infrared to green light. This requirement, plus the new operating constraints on visitors' instruments at the Keck II announced in December 1997 that exclude operation of a table-mounted spectrograph in the basement, mean that a new spectroscopic instrument that fits in the transfer module with the MAPS is needed. Our table-mounted instrument is too large for that necessarily confined space, so continuation of our participation in the MAPS program awaits funds for a new compact, portable instrument.

Acknowledgments. We appreciate the assistance and support of C. Baker, J. Chin, C. Chong, L. Coban, N. Felton, G. D. Gatewood, T. Gehrels, R.L. James, R. Laub, R. Moskitis, M. Noe, W.T. Persinger, J.V. Scotti, S. Shutes, and W.T. Verts. Grant NAG5-4598 to McMillan from NASA's Origins of Solar Systems Program has funded this effort.

\section{References}

Baranne, A., Queloz, D., Mayor, M., Adrianzyk, G., Knispel, G., Kohler, D., Lacroix, D., Meunier, J.-P., Rimbaud, G., \& Vin, A. 1996, A\&AS, 119, 373

Brown, T.M., Noyes, R.W., Nisenson, P., Korzennik, S.G., \& Horner, S. 1994, PASP, 106, 1285

Butler, R.P., \& Marcy, G.W. 1996, ApJ, 464, L153

Butler, R.P., Marcy, G.W., Williams, E., McCarthy, C., Dosanjh, P., \& Vogt, S.S. 1996, PASP, 108, 500

Cochran, W.D., \& Hatzes, A.P. 1994, Ap\&SS, 212, 281

Cochran, W.D., Hatzes, A., Marcy, G.W., \& Butler, R.P. 1997, ApJ, 483, 457

Deming, D., \& Plymate, C. 1994, ApJ, 426, 382

Dravins, D. 1978, Appl.Optics, 17, 404

Dravins, D. 1985, in Stellar Radial Velocities (IAU Coll. 88), A.G. Davis Philip \& D.W. Latham, Schenectady: L. Davis, 311

Dravins, D. 1987, A\&A, 172, 200

Dravins, D. 1992, in Proc. of ESO Workshop on High Resolution Spectroscopy with the VLT, ESO Conf. and Workshop Proc. No. 40, M.-H. Ulrich, 55 
Gatewood, G.D. 1987, AJ, 94, 213

Gatewood, G.D. 1996, BAAS, 28, 885

Gatewood, G.D., Snyder Hale, A., Snyder Hale, D.D., Persinger, W.T., McMillan, R.S., Montani, J.L., Moore, T.L., \& Perry, M.L. 1997, in Planets Beyond the Solar System and the Next Generation of Space Missions (ASP Conf. Ser., 119), D. Soderblom, San Francisco: Astron. Soc. Pacific, 41

Ge, J., Angel, J.R.P., Sandler, D.G., Shelton, J.C., McCarthy, D.W., \& Burge, J.H. 1997, Proc. SPIE, 3126, 343

Gray, D.F., \& Baliunas, S.L. 1995, ApJ, 441, 436

Gray, D.F., Baliunas, S.L., Lockwood, G.W., \& Skiff, B.A. 1996, ApJ, 465, 945

Gray, D.F., \& Livingston, W.C. 1997, ApJ, 474, 802

Hobbs, L.M. 1969, ApJ, 157, 135

Latham, D.W., Mazeh, T., Stefanik, R.P., Mayor, M., \& Burki, G. 1989, Nature, 339,38

Livingston, W. 1987, in The Role of Fine-Scale Magnetic Fields on the Structure of the Solar Atmosphere, E.-H. Schröter, M. Vázquez \& A.A. Wyller, Cambridge: Cambridge Univ. Press, 14

Livingston, W. 1991, in The Sun and Cool Stars: Activity, Magnetism, Dynamos. Lecture Notes in Physics \#380, I. Tuomin, D. Moss, \& G. Rüdigev, 246

Livingston, W. 1998, in preparation.

Marcy, G. W., \& Butler, R. P. 1996, ApJ, 464, L147

Mayor, M. \& Queloz, D. 1995, Nature, 378, 355

Mayor, M., Queloz, D., Beuzit, J.-L., Mariotti, J.-M., Naef, D., Perrier, C., \& Sivan, J.-P. 1998, in preparation.

Mazeh, T., Latham, D.W., \& Stefanik, R.P. 1996, ApJ, 466, 415

McMillan, R.S., Moore, T.L., Perry, M.L., \& Smith, P.H. 1993, ApJ, 403, 801

McMillan, R.S., Moore, T.L., Perry, M.L., \& Smith, P.H. 1994, Ap\&SS, 212, 271

McMillan, R.S., Perry, M.L., Smith, P.H., \& Merline, W.J. 1988, in Fiber Optics in Astronomy (ASP Conf. Ser., 3), S.C. Barden, San Francisco: Astron. Soc. Pacific, 237

McMillan, R.S., Smith, P.H., Frecker, J.E., Merline, W.J., \& Perry, M.L. 1985, in Stellar Radial Velocities (IAU Colloq. 88), A.G. Davis Philip \& D.W. Latham, Schenectady: L. Davis Press, 63

McMillan, R.S., Smith, P.H., Frecker, J.E., Merline, W.J., \& Perry, M.L. 1986, Proc. SPIE, 627, 2

McMillan, R.S., Smith, P.H., Perry, M.L., Moore, T.L., \& Merline, W.J. 1990 , Proc. SPIE, 1235, 601

Nordgren, T., \& Hajian, A.R. 1999, these Proceedings

Noyes, R., Jha, S., Korzennik, S., Krockenberger, M., Nisenson, P., Brown, T., Kennelly, E., \& Horner, S. 1997, ApJ, 483, L111

Perot, A., \& Fabry, C. 1899, ApJ, 9, 87 
Saar, S.H., Butler, R.P., \& Marcy, G.W. 1998, ApJ, 498, L153

Saar, S.H., \& Donahue, R.A. 1997, ApJ, 485, 319

Vaughan, J. M. 1989, The Fabry-Perot Interferometer: History, Theory, Practice, and Applications, Bristol: Adam Hilger

Vogt, S. S. 1992, in Proc. of ESO Workshop on High Resolution Spectroscopy with the VLT, ESO Conf. and Workshop Proc. No. 40, M.-H. Ulrich, 223

Wilson, O. C. 1978, ApJ, 226, 379

\section{Discussion}

Isaak: The results in tehpaper by Jiménez et al. (1986) are to be tken with a pinch of salt (my name is on it!), since over a decade ago, adequate precautions were not taken in the search for, and study of, global solar oscillations.

McMillan: O.k., thank you.

Griffin: You showed comparisons of line-bisector profiles taken with different resolving powers, i.e. with different instruments and presumably on different dates. Since the previous talk illustratedhow bisector profiles with changing degrees of magnetic activity, is not that fact perhaps confusing your statement that the different instruments show differently shaped bisectors?

Dravins: One can quantify the resolution needed to see any detailed bisector shapes. While the mere slope of the bisector (i.e. the skewness of the line) may be visible already at modest resolution, a curvature requires a minimum of three points. Since each bisector point is obtained as an average of intensity measurements in the shortward and longward flanks of the line, that implies six intensity measurements. For a photosheric line with a usable width of $9 \mathrm{~km}$ $\mathrm{s}^{-1}$, the sampling step of $1.5 \mathrm{~km} \mathrm{~s}^{-1}$ then implies a spectral resolution of $R=$ 200000 . 